\title{
openheart Cardioprotective role of zofenopril in patients with acute myocardial infarction: a pooled individual data analysis of four randomised, double- blind, controlled, prospective studies
}

\author{
Claudio Borghi, ${ }^{1}$ Stefano Omboni, ${ }^{2}$ Giorgio Reggiardo, ${ }^{3}$ Stefano Bacchelli, ${ }^{1}$ \\ Daniela Degli Esposti, ${ }^{1}$ Ettore Ambrosioni, ${ }^{1}$ on behalf of the SMILE Working Project
}

To cite: Borghi C, Omboni S, Reggiardo G, et al. Cardioprotective role of zofenopril in patients with acute myocardial infarction: a pooled individual data analysis of four randomised, double-blind, controlled, prospective studies. Open Heart 2015;2:e000220. doi:10.1136/openhrt-2014000220

Received 11 November 2014 Revised 23 June 2015 Accepted 8 July 2015
CrossMark

For numbered affiliations see end of article.

Correspondence to Professor Claudio Borghi; claudio.borghi@unibo.it

\section{ABSTRACT}

Background: Early administration of zofenopril following acute myocardial infarction (AMI) proved to be prognostically beneficial in the four individual randomised, double-blind, parallel-group, prospective SMILE (Survival of Myocardial Infarction Long-term Evaluation) studies. In the present analysis, we evaluated the cumulative efficacy of zofenopril by pooling individual data from the four SMILE studies.

Methods: 3630 patients with AMI were enrolled and treated for 6-48 weeks with zofenopril 30-60 mg/day $(n=1808)$, placebo $(n=951)$, lisinopril $5-10 \mathrm{mg} /$ day $(n=520)$ or ramipril $10 \mathrm{mg} /$ day $(\mathrm{n}=351)$. The primary study end point of this pooled analysis was set to 1 year combined occurrence of death or hospitalisation for cardiovascular (CV) causes.

Results: Occurrence of major CV outcomes was significantly reduced with zofenopril versus placebo $(-40 \%$; HR $=0.60,95 \% \mathrm{Cl} 0.49$ to $0.74 ; p=0.0001)$ and versus the other ACE inhibitors $(-23 \%$; $H R=0.77$, 0.63 to $0.95 ; p=0.015)$. The risk reduction observed under treatment with the other ACE inhibitors was nearly statistically significant $(-22 \% ; \mathrm{HR}=0.78,0.60$ to 1.02; $p=0.072$ ). The benefit of zofenopril versus placebo was already evident after the first 6 weeks of treatment $(-28 \%$; HR=0.72, 0.54 to $0.97 ; p=0.029)$, while this was not the case for the other ACE inhibitors $(-19 \% ; H R=0.81,0.57$ to $1.17 ; p=0.262)$. In this early phase of treatment, zofenopril showed a nonsignificant trend towards a larger reduction in CV events versus the other ACE inhibitors $(-11 \%$; $\mathrm{HR}=0.89,0.69$ to $1.15 ; \mathrm{p}=0.372$ ).

Conclusions: The pooled data analysis from the SMILE Programme confirms the favourable effects of zofenopril treatment in patients with post-AMI and its long-term benefit in terms of prevention of CV morbidity and mortality.

\section{INTRODUCTION}

Activation of the renin-angiotensin-aldosterone system has long been implicated in the

\section{KEY QUESTIONS}

What is already known about this subject?

- Use of ACE inhibitors has been shown to be beneficial in preventing major cardiovascular complications in several large randomised, prospective early and late intervention trials in patients with post-acute myocardial infarction (AMI).

What does this study add?

- Zofenopril is an ACE inhibitor with a proven efficacy in early treatment of AMI. Such evidence comes from four separate randomised studies of similar design comparing zofenopril with placebo, lisinopril or ramipril. A pooled analysis of these studies is provided in order to increase the robustness of the evidence of zofenopril efficacy in patients with post-AMI.

How might this impact on clinical practice?

- The results confirm the superior efficacy of zofenopril versus placebo in patients with post-AMI, and its long-term benefit in terms of prevention of major cardiovascular events. This further supports the indication to use zofenopril as well as ACE inhibitors in the treatment of AMI.

pathogenesis of acute myocardial infarction (AMI), and its blockade has been shown to be beneficial in preventing major cardiovascular $(\mathrm{CV})$ complications in several large randomised, prospective early and late intervention trials in patients with post-AMI. ${ }^{1-4}$ Accordingly, current guidelines recommend the prescription of an ACE inhibitor (ACEIs) to all patients with ST elevation anterior AMI, post-AMI left ventricular dysfunction (left ventricular ejection fraction, LVEF $<40 \%$ ), or to those who have experienced heart failure in the early phase of the AMI. ${ }^{5-7}$ ACEIs should also be given to and 
continued indefinitely in patients recovering from unstable angina or non-ST elevation AMI, or those with stable coronary heart disease (CHD), even in the absence of left ventricular dysfunction. ${ }^{7-9}$

Among the various ACEIs, zofenopril proved to be very effective in patients with $\mathrm{CHD}$ and AMI, thanks to its unique effective mechanism of action for improving blood pressure control, left ventricular function and myocardial ischaemia burden, as well as ACE inhibition. ${ }^{10}$ The double-blind, randomised, parallel-group, prospective trials of the Survival of Myocardial Infarction Long-Term Evaluation (SMILE) project, which involved more than 3600 patients with CHD, demonstrated that early AMI treatment with zofenopril may reduce mortality and morbidity, also when combined with acetylsalicylic acid, to a greater extent than lisinopril and ramipril. ${ }^{11-14}$ In addition, zofenopril has shown an interesting anti-ischaemic effect in patients with preserved left ventricular function after AMI. ${ }^{15}$

The objective of this pooled individual data analysis of the four SMILE studies was to review the cumulative efficacy of the ACEI zofenopril in the patients with CHD enrolled under the SMILE project.

\section{METHODS}

\section{Study design and population}

The four double-blind, randomised, parallel-group SMILE studies compared the efficacy and safety of zofenopril with that of placebo (SMILE-1 and SMILE-3), ${ }^{11}{ }^{15}$ lisinopril $\left(\right.$ SMILE-2) ${ }^{12}$ or ramipril (SMILE-4) ${ }^{13}$ in European men and non-pregnant women with AMI. Patients included in the studies were those with (1) an early AMI $(<24 \mathrm{~h})$, not eligible for thrombolytic therapy because of late admission to the intensive care unit or with contraindication to systemic fibrinolysis (SMILE-1); ${ }^{11}$ (2) a confirmed diagnosis of AMI and a prior thrombolytic treatment within $12 \mathrm{~h}$ of the onset of clinical symptoms of AMI (SMILE-2); ${ }^{12}$ (3) a recent AMI (within $6 \pm 1$ weeks) with preserved LVEF $(>40 \%)$, treated with a thrombolytic treatment and with ACEIs (SMILE-3); ${ }^{15}$ and (4) an early myocardial infarction $(<24 \mathrm{~h})$, either treated with thrombolysis or not, with primary percutaneous transluminal angioplasty or coronary artery by-pass graft, and with clinical and/or echocardiographic evidence of left ventricular dysfunction (SMILE-4) ${ }^{13}$ All studies were conducted in according with the Guidelines for Good Clinical Practice and the Declaration of Helsinki, and were approved by the Ethics Committee of each participating centre. Written informed consent was obtained from each patient before enrolment.

\section{Treatments}

Eligible patients were double-blinded and randomly allocated to treatment with zofenopril or comparator (placebo, lisinopril or ramipril), in addition to standard recommended therapy for AMI. No lead in observational period was foreseen prior to randomisation, except for the SMILE-4 study. In this study, eligible patients entered a 4-day open-label phase prior to randomisation and were given zofenopril according to the up-titration scheme described below. ${ }^{11}$ The initial dose of zofenopril was $7.5 \mathrm{mg}$ two times per day on day 1 and 2 , followed by $15 \mathrm{mg}$ two times per day on day 3 and 4 and $30 \mathrm{mg}$ two times per day from day 5 onward. Up-titration was allowed if systolic blood pressure remained $>100 \mathrm{~mm} \mathrm{Hg}$ and if there were no signs or symptoms of hypotension. The doses of the active comparators were up-titrated as well: up to $10 \mathrm{mg}$ once daily for lisinopril and up to $5 \mathrm{mg}$ two times per day for ramipril. Randomised treatment was continued for 6-48 weeks and patients were seen at enrolment every 1-6 months, depending on the study. For all studies, duration of treatment and follow-up periods overlapped, the only exception being represented by the SMILE-1 study. In this trial, on completion of the 6-week doubleblind treatment period, the patients stopped taking the study medication but continued treatment with their other medications for an additional 48 weeks.

\section{Statistical analysis}

This analysis was an individual patient data (IPD) analysis where the IPD were pooled. In this analysis, a one-step approach was used, the IPD were aggregated and the pooled data set was analysed using appropriate statistical methods. IPD pooled analysis improves the quality of data and produces more reliable results. Since all the four SMILE Studies provided information on fatal and non-fatal CV events, the primary study end point of this retrospective analysis was set to the 1 year combined occurrence of death or hospitalisation for $\mathrm{CV}$ causes. In the SMILE-1 study, the primary end point was the combined occurrence of death or severe congestive heart failure during the 6 weeks of treatment with zofenopril or placebo. In the SMILE-2 study, the primary end point was the 6-week occurrence of severe hypotension, either cumulative or related to study drug administration, with zofenopril or lisinopril, while occurrence of CV outcomes was a secondary end point. In the SMILE-3 study, the primary end point was the 6-month global ischaemic burden, defined as the occurrence of postinfarction angina, ischaemic abnormalities during rest or Holter ECG, or treadmill test, recurrent myocardial infarction or need for coronary revascularisation. Finally, in the SMILE-4 study, the primary end point was the 1 year rate or CV mortality or morbidity (hospitalisation for CV causes).

For the purpose of the present pooled analysis, the efficacy end point was calculated after weighing for the number of participants contributing from each study. The efficacy analysis was carried out on the full analysis set (intention-to-treat population), made up of all randomised patients treated with at least one dose of study medication and documenting at least once the measure of the primary efficacy assessment, even in case of protocol violation or premature withdrawal from the study. The safety analysis was applied to all randomised 
patients who took at least one dose of the study medication, by assessing the incidence of adverse events and changes in laboratory data or ECG during the study. The measure of safety used in this pooled analysis was the rate of drug-related adverse events expressed also as the number of drug-related adverse events divided by the person-time at risk throughout the observation period.

The baseline characteristics and the distribution of variables in the study populations and subgroups were compared using a $\chi^{2}$ test $(2 \times 4$ tables $)$ for categorical variables and an analysis of variance (between groups, F-test) for continuous variables.

HRs and $95 \%$ CIs were calculated by a Cox proportional hazard regression model in which treatment group, gender (males vs females), country, age ( $<65$ vs $\geq 65$ years $)$, body mass index $\left(<30 \mathrm{vs} \geq 30 \mathrm{~kg} / \mathrm{m}^{2}\right)$ and $\mathrm{CV}$ risk factors (yes vs no) were included as covariates. CV risk factors were defined by the presence of previous angina pectoris, previous congestive heart failure, hypercholesterolaemia requiring lipid-lowering drug, previous peripheral artery disease or previous coronary revascularisation. In order to account for the different durations of follow-up among the four studies, the relative risk of $\mathrm{CV}$ morbidity and mortality was assessed using a time-dependent Cox regression model and corresponding survival curves were drawn. Heterogeneity for the primary study end point was assessed by the $Q$ Cochrane's statistics. ${ }^{16}$ Evaluations for the primary study end point were also made by subgroups of patients according to age, gender, diabetes, hypertension and CV risk factors.

All $\mathrm{p}$ values are two-sided and the minimum level of statistical significance was set at 0.05 . Data are shown as mean \pm SD or as mean and $95 \%$ CI, or as absolute (n) and relative $(\%)$ frequencies.

\section{RESULTS}

\section{Patient population}

Three thousand six hundred and thirty patients were included in this pooled analysis: 1556 patients (43\%) were enrolled in the SMILE-1, 1024 in the SMILE-2 $(28 \%), 334$ in the SMILE-3 $(9 \%)$ and 716 in the SMILE-4 (20\%) study. Eleven European countries participated in the study, with $61 \%$ of patients recruited in Italy. One thousand eight hundred and eight patients $(50 \%)$ received zofenopril, 951 (26\%) placebo, 520 (14\%) lisinopril and $351(10 \%)$ received ramipril. Baseline characteristics for the patients included in the present pooled analysis are summarised in table 1. Some heterogeneity across the four treatment groups was observed.

\section{Long-term treatment efficacy}

As shown in figure 1A, the chance of surviving over 1 year without any major CV event was significantly higher under treatment with zofenopril than placebo
(HR and $95 \%$ CI $0.60,0.49$ to $0.74 ; \mathrm{p}=0.0001$ ), with a $40 \%$ risk reduction. The superiority of zofenopril versus placebo was evident regardless of age, gender, diabetes, hypertension or presence of CV risk factors (table 2).

The 1 year risk of mortality and morbidity was reduced by $22 \%$ under treatment with the other ACEIs versus placebo $(0.78,0.60$ to 1.02$)$, the between-group difference being nearly statistically significant $(p=0.072$; figure 1A), also when specific subgroups were examined (table 2). A larger benefit was observed with respect to placebo under lisinopril ( $\mathrm{HR}=0.63,0.47$ to 0.87$)$ than under ramipril (0.94, 0.71 to 1.26 ; figure $1 \mathrm{~B})$.

Cumulative survival rates were higher under zofenopril than under the other ACEIs (0.77, 0.63 to 0.95; $\mathrm{p}=0.015$; figure $1 \mathrm{~A}$ ), with lisinopril showing an efficacy closer to that of zofenopril (figure 1B; lisinopril vs zofenopril: $0.89,0.64$ to 1.23 ; zofenopril vs ramipril: 0.64 , 0.50 to 0.81 ).

The Q Cochran's analysis showed a moderate heterogeneity in the effect between zofenopril and the other ACEIs $\left(I^{2}=42 \%\right)$.

\section{Efficacy in the early phase of treatment}

The superiority of zofenopril versus placebo was particularly evident in the first 6 weeks of treatment, with a $28 \%$ reduction in the risk of CV mortality and morbidity (0.72, 0.54 to $0.97 ; \mathrm{p}=0.029$; figure $2 \mathrm{~A})$. Such a reduction was documented also for the other ACEIs $(0.81,0.57$ to $1.17)$, but it was not statistically significant $(p=0.262$; figure 2A). Survival rates with respect to placebo were much better under lisinopril $(0.62,0.41$ to 0.95$)$ than under ramipril (1.06, 0.71 to 1.59 ; figure $2 \mathrm{~B}$ ).

In this early phase of the study, zofenopril was as effective as the other ACEIs $(0.89,0.69$ to 1.15 ; $p=0.372$; figure $2 \mathrm{~A}$ ), and particularly with respect to lisinopril $(1.15,0.83$ to 1.60 ; figure $2 \mathrm{~B})$.

\section{Safety}

Assessment of adverse events was carried out in 3697 patients (1841 zofenopril, 956 placebo, 520 lisinopril and 380 ramipril): 328 patients receiving zofenopril (18\%), 73 placebo (8\%), 148 lisinopril (29\%) and 21 receiving ramipril $(6 \%)$ reported adverse events attributed to study treatment.

The rate of drug-related adverse events (person-time at risk) was 0.60 under zofenopril (369 events), 0.44 under placebo (102 events, $\mathrm{p}<0.001$ vs zofenopril), 2.78 under lisinopril (152 events, $\mathrm{p}<0.001$ vs zofenopril) and 0.08 under ramipril ( 16 events, $\mathrm{p}<0.001$ vs zofenopril). Thus, the rate of drug-related adverse events was lower under ramipril and higher under lisinopril, as compared to zofenopril.

In patients treated with ACEIs, a total of 60 drug-related serious adverse events occurred, of which 36 were under zofenopril ( $1.5 \%$ of total adverse events), 22 were under lisinopril $(2.2 \%)$ and 2 were under ramipril $(0.5 \%)$. 
Table 1 Demographic and clinical characteristics of the study population summarised by type of treatment

\begin{tabular}{|c|c|c|c|c|c|}
\hline & Zofenopril $(n=1808)$ & Placebo $(n=951)$ & Lisinopril $(n=520)$ & Ramipril (n=351) & p Value \\
\hline Age (years, mean+SD) & $61 \pm 11$ & $63 \pm 11$ & $59 \pm 10$ & $61 \pm 11$ & $<0.001$ \\
\hline Males (n, \%) & $1357(75)$ & $705(74)$ & $395(76)$ & $276(79)$ & 0.396 \\
\hline BMI $\left(\mathrm{kg} / \mathrm{m}^{2}\right.$, mean $\left.\pm S D\right)$ & $27 \pm 4$ & $26 \pm 4$ & $27 \pm 4$ & $28 \pm 4$ & $<0.001$ \\
\hline Major CV risk factors (n, \%) & $315(17)$ & $182(19)$ & $83(16)$ & $88(25)$ & $<0.001$ \\
\hline Diabetes (n, \%) & $1203(67)$ & $640(67)$ & $283(54)$ & $288(82)$ & $<0.001$ \\
\hline Hypertension (n, \%) & $786(45)$ & $486(52)$ & $259(51)$ & $131(40)$ & $<0.001$ \\
\hline Hypercholesterolaemia (n, \%) & $1435(79)$ & $760(80)$ & $386(74)$ & $279(80)$ & 0.054 \\
\hline
\end{tabular}

Data are shown as absolute $(n)$ and relative $(\%)$ frequencies for categorical variables and as mean $( \pm$ SD) for continuous variables. $p$ Values refer to the statistical significance of the difference across the four treatment groups $\left(\chi^{2}\right.$ test for categorical variables and ANOVA for continuous variables).

ANOVA, analysis of variance; BMI, body mass index; CV, cardiovascular.

\section{DISCUSSION}

In the present pooled analysis, we confirmed what was documented in the individual SMILE studies, namely, that treatment of patients with AMI, with or without left ventricular dysfunction, with zofenopril, effectively reduces the risk of the combined end point of $\mathrm{CV}$ death or hospitalisation for $\mathrm{CV}$ causes with respect to placebo. ${ }^{11-15}$ Interestingly, most of the beneficial effects of active drug treatment with zofenopril in our study were already evident in the first weeks following initiation of treatment, and were well maintained over time. As a matter of fact, in zofenopril-treated patients, $70 \%$ of the risk reduction was achieved in the first 6 weeks, while an additional $30 \%$ was reached at the end of the follow-up. These results are in agreement with previous data showing that treatment with ACEIs begun days to weeks after AMI improves clinical outcomes. ${ }^{17} 18$ Given the peculiar pharmacological characteristics of zofenopril, we may suggest that most of the benefit is achieved with this drug through a primary vasculoprotective and cardioprotective effect, as shown in preclinical studies in $\operatorname{animals}^{19}{ }^{20}$ and in clinical studies in humans, ${ }^{21} 22$ as well as through the prompt blockade of the deleterious effects of neurohumoral activation. ${ }^{23}$

Our study also compared the effects of zofenopril with those of two other ACEIs (lisinopril and ramipril): treatment with zofenopril reduced the chance of occurrence of the combined end point slightly more than did lisinopril or ramipril, at least in the long term. The only other available large trial assessing the efficacy of different ACEIs after AMI is a non-randomised, observational study by Hansen et $a l^{24}$ In this study, no differences were observed in the risk of mortality and reinfarction among trandolapril, ramipril, enalapril, captopril, perindopril and other ACEIs, suggesting a class effect rather than a specific activity of the single ACEI. Our results are in contrast with those of the study by Hansen et al, and do not support a class effect but, rather, support differences in the efficacy between different ACEIs. Although our data are quite consistent, being collected through
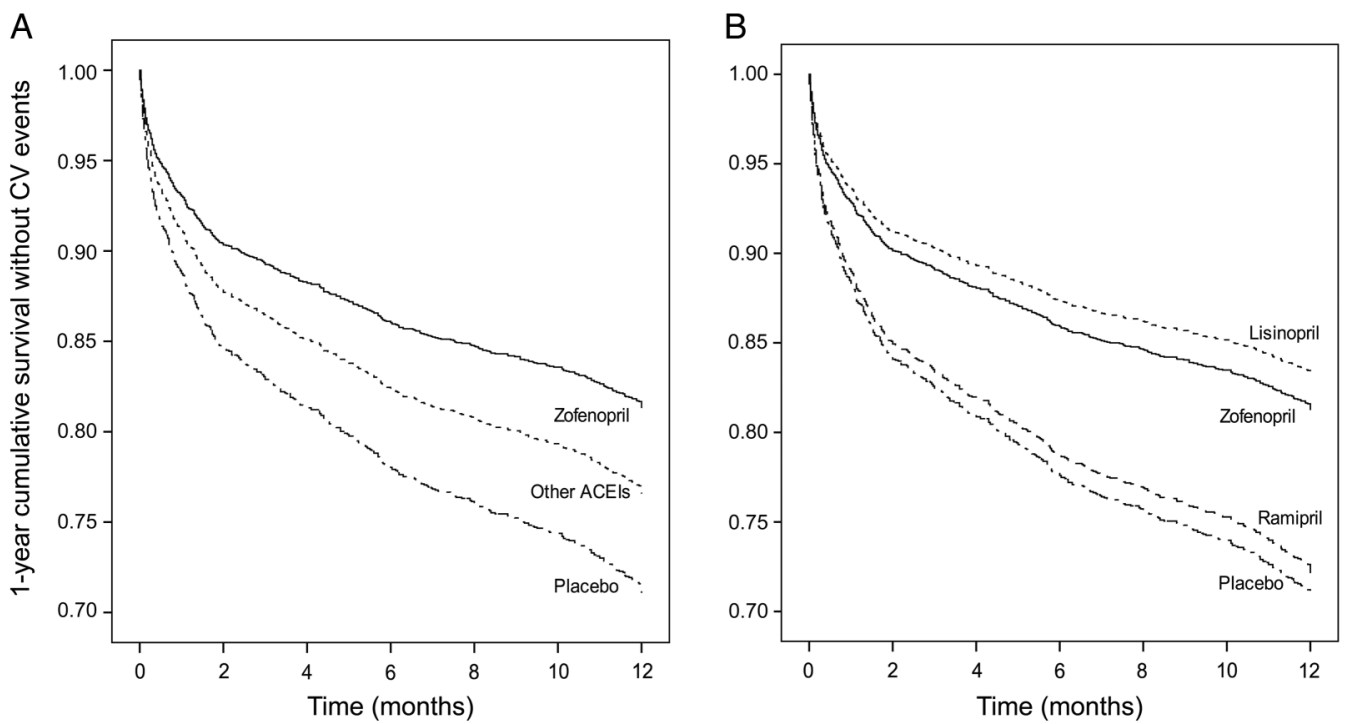

Figure 1 Cumulative survival without events during 1 year of follow-up in patients treated with zofenopril ( $n=1808)$, placebo $(n=951)$, lisinopril $(n=520)$ or ramipril $(n=351)$ in the Survival of Myocardial Infarction Long-term Evaluation (SMILE) programme. Data are shown by pooling together data obtained under lisinopril and ramipril (other ACE inhibitors, A) and separately for each treatment group $(B)$. 
Table 2 HR and 95\% Cls for 1 year cardiovascular (CV) morbidity and mortality of zofenopril versus placebo, other ACE inhibitors (ACEIs) versus placebo and zofenopril versus other ACEls

\begin{tabular}{|c|c|c|c|c|c|c|}
\hline & \multicolumn{2}{|c|}{$\begin{array}{l}\text { Zofenopril versus placebo } \\
(\mathrm{n}=2759)\end{array}$} & \multicolumn{2}{|c|}{$\begin{array}{l}\text { Other ACEls vs placebo } \\
(n=1822)\end{array}$} & \multicolumn{2}{|c|}{$\begin{array}{l}\text { Zofenopril vs other ACEls } \\
(n=2679)\end{array}$} \\
\hline & HR (95\% Cl) & p Value & HR (95\% Cl) & p Value & HR (95\% Cl) & p Value \\
\hline \multicolumn{7}{|l|}{ Age (years) } \\
\hline$\geq 65(n=1543)$ & $0.66(0.51$ to 0.85$)$ & 0.001 & 0.74 (0.51 to 1.07$)$ & 0.107 & 0.90 (0.66 to 1.21$)$ & 0.473 \\
\hline$<65(n=2084)$ & 0.53 (0.38 to 0.73$)$ & 0.0001 & 0.78 (0.52 to 1.18$)$ & 0.236 & 0.67 (0.50 to 0.90$)$ & 0.008 \\
\hline \multicolumn{7}{|l|}{ Gender } \\
\hline Males $(n=2733)$ & 0.61 (0.48 to 0.78$)$ & 0.0001 & 0.83 (0.61 to 1.15$)$ & 0.265 & 0.73 (0.58 to 0.93 ) & 0.012 \\
\hline Females $(n=897)$ & 0.60 (0.42 to 0.86$)$ & 0.005 & 0.70 (0.42 to 1.17$)$ & 0.174 & 0.86 (0.57 to 1.30$)$ & 0.479 \\
\hline \multicolumn{7}{|l|}{ Diabetes } \\
\hline Yes $(n=1216)$ & 0.61 (0.43 to 0.87 ) & 0.007 & 0.67 (0.41 to 1.12$)$ & 0.127 & 0.91 (0.61 to 1.35 ) & 0.631 \\
\hline No $(n=2414)$ & 0.60 (0.47 to 0.77$)$ & 0.0001 & 0.80 (0.58 to 1.11$)$ & 0.179 & 0.75 (0.59 to 0.95$)$ & 0.019 \\
\hline \multicolumn{7}{|l|}{ Hypertension } \\
\hline Yes $(n=1880)$ & 0.65 (0.49 to 0.86$)$ & 0.003 & 0.83 (0.57 to 1.21$)$ & 0.338 & 0.78 (0.60 to 1.01$)$ & 0.062 \\
\hline No $(n=1662)$ & 0.59 (0.44 to 0.79$)$ & 0.0001 & 0.76 (0.50 to 1.15$)$ & 0.194 & 0.77 (0.55 to 1.10$)$ & 0.150 \\
\hline \multicolumn{7}{|l|}{ CV risk factors } \\
\hline Yes (n=2962) & 0.63 (0.51 to 0.78$)$ & 0.0001 & 0.78 (0.58 to 1.05$)$ & 0.107 & 0.81 (0.64 to 1.00$)$ & 0.060 \\
\hline No $(n=668)$ & 0.45 (0.26 to 0.78$)$ & 0.004 & 0.71 (0.36 to 1.41$)$ & 0.334 & 0.62 (0.37 to 1.06$)$ & 0.081 \\
\hline
\end{tabular}

double-blind, randomised, parallel-group, prospective studies with similar designs, we cannot exclude the fact that the superiority of zofenopril might simply be related to a larger number of participants included in this group and to some heterogeneity across the studies. Future direct comparative studies should explore this aspect in detail.

Safety results confirmed that when treatment with zofenopril is initiated at low dose within the first days or weeks of onset of symptoms and signs of AMI, and up-titrated to optimal dose within a week, its tolerability is good, comparable to that observed with the reference drugs, lisinopril and ramipril, and consistent with previous clinical observations in the same field. ${ }^{25}$

\section{Study limitations}

Although the design of the four SMILE studies was very similar, there were some differences in the inclusion criteria, and treatment duration and follow-up, which might have biased the study results, particularly when direct comparisons between different active drug treatments were attempted. For instance, the SMILE-1 study included only those patients who were nonthrombolysed, the SMILE-2 and SMILE-3 included only

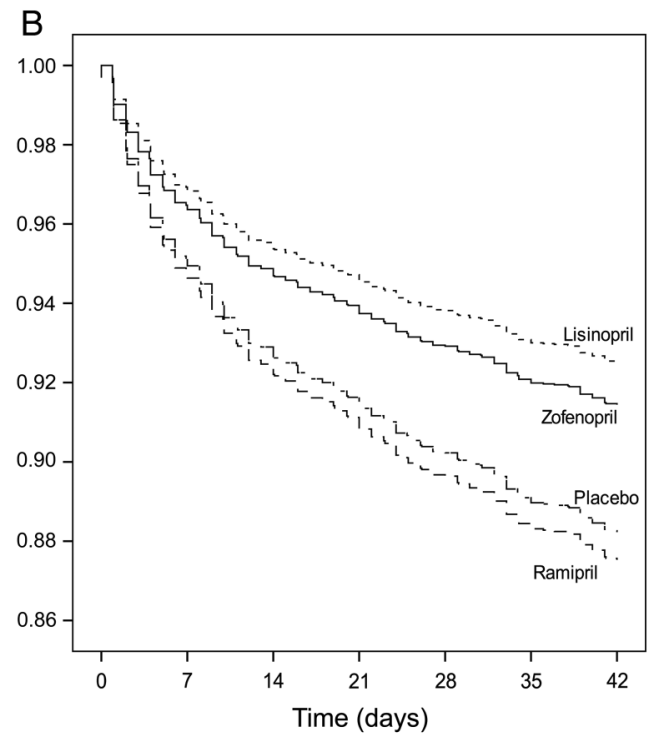

Figure 2 Cumulative survival without events during the first 42 days of treatment with zofenopril $(n=1808)$, placebo $(n=951)$, lisinopril $(n=520)$ or ramipril $(n=351)$ in the Survival of Myocardial Infarction Long-term Evaluation (SMILE) programme. Data are shown by pooling together data obtained under lisinopril and ramipril (other ACE inhibitors, A) and separately for each treatment group (B). 
thrombolysed patients, and the SMILE-4 study included both types of patients. The SMILE-3 study excluded patients with a LVEF $<40 \%$, while patients with left ventricular dysfunction were included in the SMILE-4 study. In the SMILE- 1 study, active treatments lasted 6 weeks, while observation continued for the subsequent 12 months. In the other SMILE studies, treatment duration and observation coincided, but the time interval differed. In assessments of the differences between treatments, resulting variations in baseline characteristics might have tended to decrease the sensitivity of such analyses to show interaction. However, such differences are inherent to all pooled analysis and the bias introduced into ascertainment of the average effects among the patients is usually limited in size. This is particularly true in our case, because we adjusted comparisons for confounding variables and we used individual patients' data instead of averages.

Another important study limitation concerns the interpretation of the results of the safety analysis. As a matter of fact, in the SMILE-2 study, the primary end point was a safety factor: the incidence of drug-related severe hypotension. This might explain why the proportion of patients with an adverse event was particularly high in the group of patients receiving lisinopril. Since the rate of adverse events was low in ramipril-treated patients of the SMILE-4 study, when data of these two different ACEIs were pooled together, differences were counterbalanced and thus elided.

\section{Conclusions}

The results of the pooled data analysis of the SMILE studies confirm the favourable effects of zofenopril treatment in patients with CHD. The reduction in mortality and morbidity observed in zofenopril-treated patients in comparison to placebo supports the fact that the ACE inhibition and specific pharmacological profile both contribute to the unbeaten efficacy of ACEIs in CHD. These results also strongly support the strategy of starting ACEIs early after AMI, in order to maximise their potential benefits. However, since the clinical benefits persisted during long-term treatment, this also suggests that ACEIs should not be withheld.

\author{
Author affiliations \\ ${ }^{1}$ Unit of Internal Medicine, Policlinico S Orsola, University of Bologna, \\ Bologna, Italy \\ ${ }^{2}$ Clinical Research Unit, Italian Institute of Telemedicine, Solbiate Arno, \\ Varese, Italy \\ ${ }^{3}$ Mediservice Srl, Agrate Brianza, Italy
}

Twitter Follow Stefano Omboni at @iitelemed

Acknowledgements The authors would like to thank Menarini International Operations, Luxembourg, SA, for the unconditioned support to the SMILE group.

Competing interests CB: Boheringer Ingelheim, Menarini International, Sanofi, Amgen, Takeda, Novartis, Ely Lilly and Servier Consultancy.

Ethics approval The main body was the Ethics Committee at the Hospital and University of Bologna.
Provenance and peer review Not commissioned; externally peer reviewed.

Open Access This is an Open Access article distributed in accordance with the Creative Commons Attribution Non Commercial (CC BY-NC 4.0) license, which permits others to distribute, remix, adapt, build upon this work noncommercially, and license their derivative works on different terms, provided the original work is properly cited and the use is non-commercial. See: http:// creativecommons.org/licenses/by-nc/4.0/

\section{REFERENCES}

1. Ma TK, Kam KK, Yan BP, et al. Renin-angiotensin-aldosterone system blockade for cardiovascular diseases: current status. $\mathrm{Br} J$ Pharmacol 2010;160:1273-92.

2. [No authors listed]. Indications for ACE inhibitors in the early treatment of acute myocardial infarction: systematic overview of individual data from 100,000 patients in randomized trials. ACE Inhibitor Myocardial Infarction Collaborative Group. Circulation 1998;97:2202-12.

3. Flather MD, Yusuf S, Køber L, et al. Long-term ACE-inhibitor therapy in patients with heart failure or left-ventricular dysfunction: a systematic overview of data from individual patients. ACE-Inhibitor Myocardial Infarction Collaborative Group. Lancet 2000;355:1575-81.

4. Rodrigues EJ, Eisenberg MJ, Pilote L. Effects of early and late administration of angiotensin-converting enzyme inhibitors on mortality after myocardial infarction. Am J Med 2003;115:473-9.

5. O'Gara PT, Kushner FG, Ascheim DD, et al., American College of Cardiology Foundation/American Heart Association Task Force on Practice Guidelines. 2013 ACCF/AHA guideline for the management of ST-elevation myocardial infarction: a report of the American College of Cardiology Foundation/American Heart Association Task Force on Practice Guidelines. Circulation 2013;127:e362-425.

6. Steg PG, James SK, Atar D, et al., Task Force on the management of ST-segment elevation acute myocardial infarction of the European Society of Cardiology (ESC). ESC Guidelines for the management of acute myocardial infarction in patients presenting with ST-segment elevation. Eur Heart J 2012;33:2569-619.

7. López-Sendón J, Swedberg K, McMurray J, et al., Task Force on ACE-inhibitors of the European Society of Cardiology. Expert consensus document on angiotensin converting enzyme inhibitors in cardiovascular disease. The Task Force on ACE-inhibitors of the European Society of Cardiology. Eur Heart J 2004;25:1454-70.

8. Anderson JL, Adams CD, Antman EM, et al., American College of Cardiology Foundation/American Heart Association Task Force on Practice Guidelines. 2012 ACCF/AHA focused update incorporated into the ACCF/AHA 2007 guidelines for the management of patients with unstable angina/non-ST-elevation myocardial infarction: a report of the American College of Cardiology Foundation/American Heart Association Task Force on Practice Guidelines. Circulation 2013;127:e663-828.

9. Finn SD, Gardin JM, Abrams J, et al., American College of Cardiology Foundation/American Heart Association Task Force. 2012 ACCF/AHA/ACP/AATS/PCNA/SCAI/STS guideline for the diagnosis and management of patients with stable ischemic heart disease: a report of the American College of Cardiology Foundation/ American Heart Association task force on practice guidelines, and the American College of Physicians, American Association for Thoracic Surgery, Preventive Cardiovascular Nurses Association Society for Cardiovascular Angiography and Interventions, and Society of Thoracic Surgeons. Circulation 2012;126:e354-471.

10. Ambrosioni E. Defining the role of zofenopril in the management of hypertension and ischemic heart disorders. Am J Cardiovasc Drugs 2007;7:17-24.

11. Ambrosioni E, Borghi C, Magnani B. The effect of the angiotensin-converting-enzyme inhibitor zofenopril on mortality and morbidity after anterior myocardial infarction. The Survival of Myocardial Infarction Long-Term Evaluation (SMILE) Study Investigators. N Engl J Med 1995;332:80-5.

12. Borghi C, Ambrosioni E, Survival of Myocardial Infarction Long-term Evaluation-2 Working Party. Double-blind comparison between zofenopril and lisinopril in patients with acute myocardial infarction: results of the Survival of Myocardial Infarction Long-term Evaluation-2 (SMILE-2) study. Am Heart J 2003;145:80-7.

13. Borghi C, Ambrosioni E, Novo S, et al. SMILE-4 Working Party. Comparison between zofenopril and ramipril in combination with acetylsalicylic acid in patients with left ventricular systolic dysfunction after acute myocardial infarction: results of a randomized, double-blind, parallel-group, multicenter, European study (SMILE-4). Clin Cardiol 2012;35:416-23. 
14. Franconi F, Omboni S, Ambrosioni E, et al. Effects of treatment with zofenopril in men and women with acute myocardial infarction: gender analysis of the SMILE Program. PLOS ONE 2014;9:e111558.

15. Borghi C, Ambrosioni E, Survival of Myocardial Infarction Long-term Evaluation Study Group. Effects of zofenopril on myocardial ischemia in post-myocardial infarction patients with preserved left ventricular function: the Survival of Myocardial Infarction Long-term Evaluation (SMILE)-ISCHEMIA study. Am Heart J 2007;153:445. e7-14.

16. Higgins J, Thompson S, Deeks J, et al. Statistical heterogeneity in systematic reviews of clinical trials: a critical appraisal of guidelines and practice. J Health Serv Res Policy 2002;7:51-61.

17. Sawhney JP. Angiotensin converting enzyme inhibitors in acute myocardial infarction - a review. Indian Heart $J$ 2011;63:71-8.

18. Donnelly R, Manning G. Angiotensin-converting enzyme inhibitors and coronary heart disease prevention. $J$ Renin Angiotensin Aldosterone Syst 2007;8:13-22.

19. Evangelista S, Manzini S. Antioxidant and cardioprotective properties of the sulphydryl angiotensin-converting enzyme inhibitor zofenopril. J Int Med Res 2005;33:42-54.
20. Bozcali E, Dedeoglu DB, Karpuz V, et al. Cardioprotective effects of zofenopril, enalapril and valsartan against ischaemia/reperfusion injury as well as doxorubicin cardiotoxicity. Acta Cardiol 2012;67:87-96.

21. Napoli C, Sica V, de Nigris F, et al. Sulfhydryl angiotensin-converting enzyme inhibition induces sustained reduction of systemic oxidative stress and improves the nitric oxide pathway in patients with essential hypertension. Am Heart J 2004;148:e5.

22. Cacciatore F, Bruzzese G, Vitale DF, et al. Effects of ACE inhibition on circulating endothelial progenitor cells, vascular damage, and oxidative stress in hypertensive patients. Eur J Clin Pharmacol 2011;67:877-83.

23. Borghi C, Bacchelli S, Degli Esposti D. Long-term clinical experience with zofenopril. Expert Rev Cardiovasc Ther 2012;10:973-82.

24. Hansen ML, Gislason GH, Køber L, et al. Different angiotensinconverting enzyme inhibitors have similar clinical efficacy after myocardial infarction. Br J Clin Pharmacol 2008;65:217-23.

25. Agustí A, Bonet S, Arnau JM, et al. Adverse effects of ACE inhibitors in patients with chronic heart failure and/or ventricular dysfunction : meta-analysis of randomised clinical trials. Drug Saf 2003;26:895-908 